\title{
Correction to: Leptin and cardiovascular health in high body mass index
}

\author{
Chee H. Liew ${ }^{1,2,3}$ - Wee H. Sim ${ }^{4}$ - Ernest Z. Low ${ }^{5}$ • Bernard C. M. Chan ${ }^{6}$ \\ Published online: 6 August 2020 \\ (C) Royal Academy of Medicine in Ireland 2020
}

\section{Correction to: Irish Journal of Medical Science (1971 -)} https://doi.org/10.1007/s11845-020-02288-2

The above article was published online with an error. In the second last paragraph, the " $\mathrm{kg} / \mathrm{m}$ " in two places (the last sentence) should be " $\mathrm{kg} / \mathrm{m}^{2}$ ". The superscript 2 is missing in two places. The original article has been corrected.

The online version of the original article can be found at https://doi.org/ $10.1007 / \mathrm{s} 11845-020-02288-2$

\section{Chee H. Liew}

cliew@tcd.ie

1 School of Medicine, National University of Ireland Galway, Galway, Ireland

2 National Institute for Prevention and Cardiovascular Health, Galway, Ireland

3 School of Medicine, Trinity College Dublin, Dublin, Ireland

4 Department of Medicine, University Hospital Limerick, Limerick, Ireland

5 Department of Geriatric Medicine, Letterkenny University Hospital, Letterkenny, Ireland

6 Department of General Surgery, Our Lady of Lourdes Hospital, Drogheda, Ireland 